Article

\title{
Understanding the development of self-efficacy for physical activity engagement in men after myocardial infarction: A preliminary qualitative study
}

\author{
Harlinna binti Abu, ${ }^{1}$ Salizar binti Mohamed Ludin, ${ }^{1}$ Siti Noorkhairina binti Sowtali \\ ${ }^{1}$ Department of Critical Care Nursing, Kulliyyah of Nursing, International Islamic University Malaysia, Jalan \\ Sultan Ahmad Shah, Pahang; ${ }^{2}$ Department of Professional Nursing Studies, Kulliyyah of Nursing, International \\ Islamic University Malaysia, Jalan Sultan Ahmad Shah, Pahang, Malaysia
}

\begin{abstract}
Background: This preliminary finding from a qualitative study examined the process of self-efficacy for the development of physical activity (PA) during myocardial infarction (MI) after recovery. A combination of healthy behaviors, including physical activity is the secondary prevention recommended to reduce the risk of recurrent MI. This study aims to understand how self-efficacy for physical activity is developed in a patient after MI by examining their perceptions and personal adherence to physical activity.

Design and Methods: This was a qualitative study and data was collected through semi-structured in-depth recorded phone interviews with eight Malay male participants. They were screened using a questionnaire and participants that met the inclusion criteria were interviewed, and were admitted to National Heart Centre, Malaysia between January to June 2019 diagnosed with MI. The data collected were analyzed using NVivo 12 software and thematic analysis was applied.

Results: Four preliminary themes emerged from the study: i) beliefs in physical activity; ii) healthy lifestyle: new normal or same old habit; iii) factors determining participation in PA; and iv) physical activity adherence strategies.

Conclusions: The results of the studies showed that participants understand the need to maintain physical activity, which helps to maintain a healthy life after MI and prevent recurrent infarction. Strategies for developing self-efficacy for physical activity were also discussed. The need to understand that maintaining physical activity as well as adopting a new normal of healthy habit after MI is crucial in order to maintain the health and prevent recurrence of MI.
\end{abstract}

\section{Introduction}

A combination of healthy behaviors, including physical activity (PA) is the secondary prevention recommended for reducing the risk of recurrent myocardial infarction (MI). ${ }^{1} \mathrm{PA}$, as defined by World Health Organization is any body movement produced by skeletal muscles that require energy expenditure. This includes activities carried out while working, playing, carrying out household chores, traveling and engaging in recreational pursuits. ${ }^{2}$ Exercise is a subcategory of PA that is planned, structured, repetitive, and aims to improve or maintain one or more components of physical fitness. $^{2}$

Self-efficacy is a strong, effective coping strategy displayed by an individual. ${ }^{3}$ It is believed that it is the determinant factor in maintaining PA. Self-efficacy is the most consistent correlate to the structured PA interventions. ${ }^{4,5}$ A number of studies reported out that self-efficacy was improved by the impact of intervention or cardiac rehabilitation. ${ }^{6-12}$ However, these studies focused on patients with coronary heart disease (CHD) and acute coronary syndrome (ACS).

According the study conducted in Jordan, which measure PA levels and self-efficacy during early recovery after MI showed cardiac self-efficacy scores improved significantly after two weeks and six weeks from baseline measurement. ${ }^{13}$ However, there were no significant differences in PA and no significant relationship between cardiac self-efficacy and PA and both times follow up measurement.

A systematic review with meta-analysis focusing on the development of self-efficacy for PA phenomenon among healthy adults. In addition, 27 studies were reviewed and discovered a small but significant relationship between the PA intervention and the changes in self-efficacy. ${ }^{14}$ However, few understandings about the insights into the development of self-efficacy in PA among MI patients. This is a preliminary study to understand how self-efficacy for PA developed in a patient after MI by examining their perceptions and personal adherence to PA.

\section{Design and Methods}

This study was conducted among myocardial infarction (MI) patient admitted at the National Heart Institute, Malaysia (NHI).

\section{Significance for public health}

The study result contributes to the knowledge base by providing evidence that influence practice and policy initiatives individuals recovering from myocardial infarction. Self-efficacy is a modifiable factor, and therefore this qualitative study provides detailed information to increase involvement in physical activity and maintaining lifestyle. The ultimate goal of this study is to reduce the risk of recurrence of myocardial infarction due to physical inactivity, and eventually improving the quality of life of myocardial infarction survivors. 
The list of patients who had been initiated with the ST-elevation MI clinical pathway from January 2019 to July 2019 was obtained from the center. The study was carried in October 2019, while the participants were discharged to their various home at least three months from the data collection period. The list was shortlisted for Malay male participants, age less than 65 years. Ethical approval was sought from the National Heart Institute Ethical Committees and the IIUM Ethics Committee was notified.

The potential participants were contacted for the first time and were asked if they have been diagnosed with metastatic cancer, end-stage heart or renal disease, do not understand Malay or English language, diagnosed with heart failure, and bedridden or wheelchair-bound. If this was not one of the exclusion criteria, participants were asked if they would agree to participate in the study and oral consent was obtained. The primary assessment was a set of questionnaires that consist of demographic data, medical history, and the International Physical Activity Questionnaire (IPAQ), and the IPAQ is a validated self-report instrument adapted from the Seven-day Physical Activity Recall Questionnaire, to assess PA levels in participants. PA levels are quantified as total energy consumption in metabolic equivalent times (MET) $\mathrm{min} /$ week and the total number of PA minutes per week for each participant.

The questions were read to the participant and the researcher marked the answer given. An appointment for the second phone call was made based on the participant's preference and the participants were asked to be alone or quiet during the phone call. The second data collection was carried out by phone call based on the appointment time. The phone call is a semi-structured in-depth recorded phone interview that lasted approximately 30-45 min. The structure of the interview guide was based on the development of self-efficacy related to the four main sources of influence; i) performance accomplishments/ mastery experience; ii) vicarious experience; iii) social persuasion; and iv) physiological and emotional states. ${ }^{15}$

To ensure the reliability of the study, once the data analysis is complete, a review of the members will be carried out by providing coded transcripts and giving participants the opportunity to reflect and ensure the interpretation. Affirmation skill is achieved by transcribing the narratives in the form of verbatim to explain the themes and subthemes acquired. The researcher discussed the codes and themes that emerged with the team members.

\section{Results and Discussions}

The interviews were transcribed verbatim, and the researcher filled in the analysis line by line to suggest the concept, properties, and dimensions of the data. This is a preliminary result, therefore the themes and sub-themes emerged was based on the open coding, the first level of the systematic data analysis. ${ }^{16}$ There were right about Eight Malaysia male participants that take part in this preliminary study, and the demographic information, and smoking status are described in Table 1. The age ranged from 28 to 61 years old. All were married and lived with their spouse and children except P5, that had divorced and lived with his children only. Table 2 shows the physical activity they usually participated in during their leisure time, and the IPAQ score and the PA level categories. The IPAQ score from the participants may not replicate the activity reported by the participants through the interview. The IPAQ was the activity accomplished by the participant for the last seven days

Table 1. The demographic data and medical history of participant,

\begin{tabular}{|c|c|c|c|c|c|c|c|c|c|c|}
\hline $\begin{array}{l}\text { ID } \\
\text { No. }\end{array}$ & Age & Education & $\begin{array}{l}\text { Marital } \\
\text { status }\end{array}$ & Occupation & $\begin{array}{l}\text { Live } \\
\text { with }\end{array}$ & $\begin{array}{l}\text { Medical } \\
\text { history }\end{array}$ & Family history & $\begin{array}{l}\text { Diagnosis } \\
\text { date }\end{array}$ & LOS & Smoking status \\
\hline Pl & 28 & Degree & Married & Working & Spouse only & None & HPT, stroke, HD & 07/02/19 & 6 & Quit (February 2019) \\
\hline P2 & 47 & Secondary school & Married & Working & Spouse and children & None & HD & $11 / 03 / 19$ & 3 & Yes (10 cig/day) \\
\hline P3 & 58 & Secondary school & Married & Retired & Spouse and children & DM, HPT, HC, HD & NONE & 27/06/19 & 5 & Quit (June 2019) \\
\hline P4 & 61 & Secondary school & Married & Retired & Spouse and children & DM, HPT & DM & 03/07/19 & 16 & Quit (July 2019) \\
\hline P5 & 47 & Secondary school & Widower & Working & Children only & DM, HPT, HC & DM, HPT & 01/04/19 & 5 & Never \\
\hline $\mathrm{P} 6$ & 41 & Secondary school & Married & Working & Spouse and children & HPT & $\mathrm{DM}, \mathrm{HD}$ & 18/05/19 & 4 & Never \\
\hline P7 & 44 & Secondary school & Married & Working & Spouse and children & HPT & DM, HPT, HD & 17/06/19 & 16 & Yes (4 cig/day) \\
\hline P8 & 50 & Secondary school & Married & Working & Spouse and children & None & None & 27/07/19 & 7 & Quit (July 2019) \\
\hline
\end{tabular}

DM, diabetes mellitus; HPT, hypertension; HC, high cholesterol; HD, heart disease, LOS, length of stay; cig, cigarettes.

Table 2. Types of physical activity and IPAQ score.

\begin{tabular}{|c|c|c|c|c|c|c|}
\hline ID No. & Physical activity & IPAC & score (MET minute & a week) & & PA category \\
\hline & & Vigorous activity & Moderate activity & Walking & Total & \\
\hline P1 & Walking & 0 & 0 & 462 & 462 & Low \\
\hline P2 & Walking, work-related activity, cycling & 0 & 1200 & 0 & 1200 & Moderate \\
\hline P3 & Walking, house chores & 0 & 0 & 264 & 264 & Low \\
\hline P4 & Gardening & 0 & 0 & 297 & 297 & Low \\
\hline P5 & Walking, house chores, gardening, leisure badminton & 0 & 240 & 198 & 438 & Low \\
\hline P6 & Work-related activity & 0 & 4800 & 66 & 4866 & High \\
\hline P7 & House chores & 840 & 6000 & 66 & 6906 & High \\
\hline P8 & Gardening & 2880 & 0 & 247.5 & 3127.5 & High \\
\hline
\end{tabular}

IPAQ, International Physical Activity Questionnaire; MET, metabolic equivalent. 
and the type of activities were categorized as vigorous activity, moderate activity and walking. While, in the interview, the participants were asked regarding the usual activity carried out during their free time or routine on PA after they had the MI.

Most participants reported that walking was their main PA, and the participants understand that the walking activity should be at least $10 \mathrm{~min}$ in each session. The other PA discussed were daily chores such as mopping and sweeping the floor, gardening, workrelated activity, cycling and playing badminton. A phone interview was carried out in order to understand the development of self-efficacy for PA in Malay men after MI. Four themes emerged from the initial open coding by analyzing the transcript line by line.

\section{Findings from interviews}

The preliminary analysis of the data from the interview showed a range of key themes. The preliminary findings guided the researcher on the subsequent interviews in order to explore more than what had been revealed.

\section{Beliefs in physical activity}

When the participants were asked regarding their thoughts on PA and why are they are participating, they reported that PA can improve their health and wellbeing:

"If you have high blood pressure, you must exercise, that's all. There are no other medicines. Hospital remedies are necessary but more to exercise. If we have diabetes, high blood pressure, cholesterol, you should exercise. I have experienced it all. Because before I had a heart attack, I don't have high blood pressure or high cholesterol. I do garden work, don't I?' (P4).

The same participant reported that before he had MI, he knew that he had to take care of his heart by being active because he normally smoke:

"Before this, (MI) I do garden work. Every day I sweat a lot. That's why I don't have high blood pressure, cholesterol. So, when I have this (MI) I am curious. I know the fact that I smoke, I have to take care of my heart, that's why I have to exercise, do heavy work" (P4).

In addition to the general health, PA have also been reported to improve their emotional health where the term used was "relieve the stress": "firstly, to make me sweat. Another one is to spend time with children. We want to have fun, we want to have leisure time, we want to destress. We always keep thinking (stress), if we have leisure time, at least it will help us to be better............ Outdoor activities, sports, help us become more active both mentally and physically" (P5).

Most of the participants also reported that they enjoyed the feeling of been energized after PA and describe the feeling by having "bodily pain" if they are inactive (P1, P2, P3, P4, P8). P2 describes the feeling by stating:

"To me, physical activity, other than making my body healthier, we feel like we don't have any pain when we are sweating. We feel if we are not feeling well, we will try to do something (physical activity) that can make us sweat".

\section{Healthy lifestyle. New normal or same old habit}

Most of the participants reported important health behaviors including food habits, medication adherence, and quitting smoking. The type of food intake varies, and the participants reported that they are advice on the type of healthy food by a nutritionist from diet counselling before been discharged home and switching to a healthier food intake. P8 states that "I have changed my eating habit; I don't take oily food... I drink milk.... I drink milk two times a day. Morning and night". However, not all participants adhered to the advice for healthy food habit despite the information they have. P2 states "About the eating habit, frankly speaking, I eat what I wanted...".

It has been claimed that taking medication regularly is a new habit that needs to be normalized, especially for participants that have never had health problems before. Five participants adhered to the medication prescribed and determined to get healthy, while two other participants claimed they took medicines but not as advised. One participant honestly claimed that he is not taking the prescribed medicines anymore.

"For me, to all people that have this like me, the heart attack I think, if you can, like you (interviewer) have said, you need to focus on what the doctors have informed you. Like, take the medicines, things that we are not supposed to take, don't take it, that it is my suggestion. Though the things I've said I am not doing it, in front of the doctor you said yes, at his back you said no isn't it. So, you (interviewer) also know right, we are human being...... ................ I, frankly speaking, I don't take the medicines" (P2).

Meanwhile, $\mathrm{P} 1$ developed a new routine of taking medications as a reminder for him to stay healthy. P1 states:

"From the morning you know that you need to take medicines, so you don't take nasi lemak. Better you take oat, isn't it? You can have nasi lemak, but it is better you have oat. We know that when we do research (reading), oats can reduce cholesterol. High cholesterol can cause heart problems, so eat oats, dates...and other healthy food".

Four out of the eight participants quit smoking on the day they were admitted, while two participants never smoke, and the other two participants reported they tried to reduce the number of cigarettes but unable to quit smoking for good: "God's willing, I have reduced (smoking) right now. I can't stop straight away like that, isn't it?"' (P2).

\section{Factors determining participation in PA}

Participants explained the reason they participated or not in PA through three aspects including intrapersonal factors, interpersonal factors and knowledge that the participants had after the AMI. Intrapersonal factors were reasons that solely arose from the participants' ego. Most of the participants discussed their own willingness and attitude to participate in the PA. Some stated their reasons for not participating in the PA was "lazy".

P2 states: "It is your own self. No one can force unless yourself. It is all about " $M$ " sickness (refer to "Malas", Malay word of lazy)".

Fear was also discussed by the participants, where it was described as fear of recurrent, which is the reason they participated in PA.

"This means that it is our efforts not to have it (MI) again. Therefore, it is necessary to participate in the physical activity not because you were forced to do, but because of your own consciousness, isn't it?" (P1)

While fear of pain discouraged the participants from participating in PA:

"So, that thing (pain) is the one that causes me like... ha... you can feel the pain even if you do nothing, how is it if the 
heart is pumping fast? The main issue is not that I don't want to do physical activity. Even if I am lying down while watching television, I can suddenly feel pain, $m m m$.... when I am relaxing and calm and do nothing... suddenly it (pain) comes. That is something that leaves as a question to me" (P1).

Some participants discussed their physical factors contributed to the PA.

"I don't have a problem if walking only, because my job needs me to walk, settle inside and out (a premise) about 30 minutes and I don't have a problem with that. If running maybe can make me exhausted, because my body weight is more than 100 $(\mathrm{kg})$ " (P6).

Interpersonal factors were related to the external prompts that influence PA participation or non-participation. Some participants explained comparing themselves with other people is a source of encouragement to participate in PA but at the same time they feel like a failure if they did not manage to comply.

"Yes, because I am 28 years old, I am still young. That means, there were people that older than me, my seniors at school before, still play sports with me (before MI). But when we have a gathering together, they will say to me, "pity you, you cannot play (rugby) anymore like before". My coach who is older than me can still play, I am only 28 years old struggle to come back (for rugby)" (P1).

Some participants described others' experience as something inspiring them to do PA.

"I do not know him closely. I know it through my friend that own a gymnasium. He had a friend that had a heart attack before but, my friend told me because he is one of his customers (at the gymnasium), he always comes to the gym and workout, and he can manage his heart problem" (P5).

While some others had a negative experience that they encounter

"There's something happened, where my wife's friend, a male, had a heart attack, then he went to the hospital. He went to hospital and then he didn't do that (angiogram/angioplasty) yet because of the cost. He went back home, and in two days at home, when he did an exercise on that day, he passed away. Maybe he is doing a vigorous exercise, that is why he cannot (cope)" (P7).

Personal and family commitment during their free time also affect participation in PA. Some participants explained that they were already busy during the weekdays with their job, and weekdays were for their family. Some felt "selfish" if they spend their free time on their own activity instead of family. "Then at the weekend, I need to go to my hometown. When you go back hometown, there is no time to do physical activity, or jogging, or exercise, you know" (P1).

Most participants had obtained some knowledge after AMI, either from the healthcare provider or personal acquisition. Some were able to apply the knowledge in their life after MI.

"When I have free time, wander at the office, and I saw the car (employer's car) is dirty, I will wipe it. That is one of the exercises, right? My boss' car is big, he got Harrier, so when you wipe the car especially during the rainy season, will make you sweat isn 't' it? That is the type of activity at the office if I have nothing to do...

. Because I know this heart attack thing people say as the silent killer, isn't it?" (P2).
Some had the knowledge but misunderstood it and practiced it wrongly, the way they understand.

"Like if you have high blood sugar, for example, I have it, that's why I do heavy work, sweating every day, so it can control my blood sugar. Ha....so for example, if the recommendation is to take the medicines two times per day, I will take once only, the other, I do the work (physical activity)" (P4).

Some participants understand the knowledge but understand their attitude towards PA participation.

"That is (physical activity) something that I already understand, but I am not doing it yet. Meaning that there were few nurses from the hospital informed me to be positive only. So, if you can be positive, you can stay positive. I understand that it's just that for me to reach that stage still in my planning" (P1).

\section{Physical activity adherence strategies}

The participants also discussed how they can be committed to PA after the MI. The participants shared their experiences while doing exercise by knowing and understanding their body limits.

"When you know how your body can go, you can try. When you feel your body cannot cope, don't push it. Usually, when I run, then I feel tired, I will walk, I will not stop straight away. I am an exarmy, I know, I have done physical stuff before, right? After running, you cannot stop (suddenly), we have to walk or warm-up or do what necessary, but do not stop abruptly" (P2). The participants also were asked if they knew anyone that had a heart attack but leads an active lifestyle. The way some of the participants described the person showed their interest and was inspired by the way the 'role model' manages the illness and was able to stay active. "Yeah, I have one block and I felt hard to do physical activity, but he had three blocks, but he can jog every day" (P1).

Companionship was another sub-theme that emerged from a few participants that reported they carried out the PA accompanied by others, especially family members. Even if they have no companion, they made sure to bring the cell phone for safety purposes. P2 states; "Yes, the two of us (wife) walking or sometimes with my brother-in-law, with my wife, we normally ride the bicycle"

The most difficult thing for PA adherence is to create a routine. Some participants voiced out that they need to plan for a routine for them to bond with in order to maintain the PA and fitness. However, a participant had mentioned the word "planning the routine" a few times, but not making it happen, and did not adhered to PA. It starts with the idea that you have to do it first and then only the routine that is set, not just the planning.

When asked about any physical activity routine that they usually do, P1 states:

"Yes, that is already in my planning, because I just got married a month ago, and moved to Seremban (a new place). Since I am in a new place, I had planned for my physical activity.... and I will try to arrange and create a routine of doing physical activity for half an hour, every evening".

The healthcare provider (HCP) plays an important role in maintaining PA in MI patients. The most important moment is before the patient is discharged home. If the HCP did not give meaningful advice during this period, the patient may end up default the follow-up treatment and continue the unhealthy lifestyle. Most of the participants voiced out that they follow the advice received from the HCP before they were discharged from 
the hospital. "I tried because the doctor said I have to do exercise, physical activities that people say don't stay still, just sit and thinking about the negative thoughts" (P5).

All the strategies may not work if the MI patient is not selfmotivation. Most of the participants said that they need to do it their own way and by determination, which cannot depend on others except oneself. The role of HCPs is not only to give medical information and advice related to their illness, but to create selfmotivation in MI patients. Some patients described self-motivation as one of the factors that leads to adhering to PA. P2 states; "For me, friends are not a problem. If I want to do (physical activity), I just do it. If I want to do it, I have nothing to do with waiting for friends, or place to do". It was the same with P7 that stated "If you want to depend on anybody then it will be difficult. It has to be you yourself".

Two meta-analysis studies that dealt with the development of self-efficacy were reviewed. The first aims to determine the relationship between the intervention and the change in self-efficacy in 27 of the same research. ${ }^{14}$ The participants were healthy adults and the intervention intended to increase self-efficacy for PA. While another study aims to review how interventions affect self-efficacy then leading to behavior change in PA. ${ }^{17}$ The first meta-analysis reported that the intervention associated with higher levels of selfefficacy was feedback on past and other's performance and vicarious experience. ${ }^{14}$ While the subsequent meta-analysis showed that action planning, reinforcing efforts and provision of instructions associated with the increment of self-efficacy and PA. ${ }^{17}$

Action planning is defined as "detailed and accurate planning of when, where, and how certain behaviors are performed". ${ }^{17}$ The participants in the studies reported they created routines for schedule PA and with routines, the participants felt they can prioritize PA into their daily activities. This study was able to create a routine as a sub-theme of the strategies of PA adherence. A participant reported routine of taking medications reminded him taking part in PA. While few participants admitted that they had planned for the routine except that they are not doing it yet. This shows that action planning is not enough if it is not applied.

These two meta-analysis studies also highlighted the experience of substitution as an intervention, which is associated with greater personal effectiveness. This study showed the initial subtheme of a role model as the strategies of PA adherence. Furthermore, compared with significant and experience of others, it is categorized under interpersonal factors determining the PA adherence. Participants that had a bad or horror experience of others such as death during PA, may be influenced to adhere to PA or being afraid to participate in PA.

A study reported that self-efficacy and outcome expectancies directly influenced PA adherence and self-efficacy influenced outcome expectancies. ${ }^{18}$ Outcome expectancies are defined as a person's belief that performing a behavior will result in specific consequences. ${ }^{19}$ In this study context, it reflects that when the post-MI patients believed that PA engagement have benefits in anyways, including improvement of physical and emotional health, reducing harm from the other unhealthy lifestyle and the enjoyment of feeling fresh and energized, they will influence their PA adherence and subsequently developed their self-efficacy for PA. Participants explained in detail how they believe joining PA can improve their health. PA is highlighted to improve physiological health and reducing the progression of the chronic state that includes cardiovascular disease, cerebral vascular accident, type- 2 diabetes mellitus, hypertension and various cancers. ${ }^{20}$

The positive influence on mental health is also acknowledged by the participant. A participant stated that they feel positive, and reduce stress with PA. This is in line with an in-depth theoretical study that reported that PA helps and makes you feel good about depression. ${ }^{21}$ The self-reinforcing helped to overcome the barriers to being physically active, which in this study was related to the development of PA self-efficacy.

The second theme that emerged was lifestyle behaviors. Selfefficacy is a fundamental component of the social cognitive theory and addresses the role of an individual's confidence that they can carry out the behavior required. According to a study, all behavioral changes are mediated by self-efficacy, where a person with low self-efficacy is less likely to have the confidence to carry out a new health behavior or try to change an embedded behavior. ${ }^{15}$ This theme can be discussed together with the sub-theme of knowledge and the role of HCPs.

Early educational intervention from the HCP before the patient is discharged home showed a significant improvement in PA and other healthy lifestyle behaviors include healthy eating habit and smoking. ${ }^{22-24}$ The follow-up measurement was between three to 12 months post-discharge.

There were other studies that inculcate the educational intervention before the patient is discharged that measured self-efficacy through Cardiac Self-efficacy Scale for AMI patients. ${ }^{25,26}$ Both interventions were implemented by nurses, and a study reported that cardiac self-efficacy scale in the intervention group after a four-week follow-up was significantly different from that in the control group. ${ }^{26}$ While the other study showed no significant difference between before discharge and three months after discharge. ${ }^{25}$ This may be due to the different design of the study used, which was the randomized controlled trial and longitudinal study. ${ }^{25,26}$

The role of HCPs was crucial in preparing MI patients with knowledge and self-efficacy before they were discharged home. The sub-themes explained i.e. lifestyle behavior, knowledge and role of HCPs may or may not be related to each other. The relationship between the emerged themes will be analyzed in the next stage of grounded theory principles, that will provide a step towards the development of the conceptual model or theory.

\section{Conclusion}

The results showed that participants understand the need for PA compliance to maintain a healthy life after a myocardial infarction and to prevent recurring heart attacks. Participants voiced out the benefits of PA that they believed can improve their physical and emotional health, and strategy for developing self-efficacy for PA was also discussed. Although this article only showed initial themes that emerged from eight participants, the next phase of this study should be able to picture a conceptual model that can represent how an individual recovering from MI develops self-efficacy for PA and can guide the secondary prevention intervention. They need to understand that adhering to PAs, as well as adopting other behaviors, are critical to a healthy lifestyle in order to reduce harm to their health and life. 
Correspondence: Salizar binti Mohamed Ludin, Department of Critical Care Nursing, Kulliyyah of Nursing, International Islamic University Malaysia, Jalan Sultan Ahmad Shah, 25200 Kuantan, Pahang.

Tel. +60.9.5707201 - Fax: +60.9.5133615.

E-mail: msalizar@iium.edu.my

Key words: Physical activity; myocardial infarction; self-efficacy; physical activity; self-efficacy.

Acknowledgment: The author expresses profound gratitude to Kuliyyah of Nursing, International Islamic University for their kindness and support during this study.

Contributions: All authors contributed equally to this article. HA conducted the study, collecting and analyzing data, drafting and was in charge of the manuscript. SML and SNS served as supervisors, final approval of the interpreting data and reviewed the final article

Conflict of interests: The author declares no potential conflict of interest.

Ethical approval: This study has been approved by Research Ethical Committees of the National Heart Institute, Malaysia.

Conference presentation: Part of this study was presented at the $1^{\text {st }}$ International Nursing and Health Sciences Symposium, November $13^{\text {th }}$ to $15^{\text {th }} 2020$, Brawijaya University, Malang, Indonesia.

Received for publication: 20 January 2021.

Accepted for publication: 15 March 2021.

(c) Copyright: the Author(s), 2021

Licensee PAGEPress, Italy

Journal of Public Health Research 2021;10:2206

doi:10.4081/jphr.2021.2206

This work is licensed under a Creative Commons Attribution NonCommercial 4.0 License (CC BY-NC 4.0).

\section{References}

1. Malaysian Ministry of Health [Internet]. Primary \& Secondary Prevention of Cardiovascular Disease 2017. Accessed: 2020 Nov 12. Available from: https:/www.malaysianheart. org $/ \mathrm{p}=\mathrm{cpg} \& \mathrm{a}=1171$

2. WHO. Physical activity. 2018 Accessed: 2020 Nov 12. Available from: https://www.who.int/news-room/factsheets/detail/physical-activity

3. Bandura A. Self-efficacy: Toward a unifying theory of behavioural change. Adv Behav Res Ther 1978;1:139-61.

4. McAuley E, Morris KS, Motl RW, et al. Long-term follow-up of physical activity behaviour in older adults. Heal Psychol 2007;26:375-80

5. Perkins JM, Multhaup KS, Perkins HW, et al. Self-efficacy and participation in physical and social activity among older adults in Spain and the United States. Gerontologist 2008;48:51-8.

6. Alsaleh E, Windle R, Blake H. Behavioural intervention to increase physical activity in adults with coronary heart disease in Jordan. BMC Public Health 2016;16:643.

7. Houle J, Doyon O, Vadeboncoeur N, et al. Innovative program to increase physical activity following an acute coronary syndrome: Randomized controlled trial. Patient Educ Couns 2011;85:e237-44.

8. Howarter AD, Bennett KK, Barber CE, et al. Exercise self-efficacy and symptoms of depression after cardiac rehabilitation. J
Cardiovasc Nurs 2014;29:168-77.

9. Izawa KP, Watanabe S, Omiya K, et al. Effect of the self-monitoring approach on exercise maintenance during cardiac rehabilitation: A randomized, controlled trial. Am J Phys Med Rehabil 2005;84:313-21.

10. Luszczynska A, Sutton S. Physical activity after cardiac rehabilitation: Evidence that different types of self-efficacy are important in maintainers and relapsers. Rehabl Psychol 2006;51:314-21.

11. Reid RD, Morrin LI, Pipe AL, et al. Determinants of physical activity after hospitalization for coronary artery disease: the Tracking Exercise After Cardiac Hospitalization (TEACH) Study. Eur J Cardiovasc Prev Rehabil 2006;13:529-37.

12. Van Sluijs EMF, Van Poppel MNM, Twisk JWR, et al. Physical activity measurements affected participants' behaviour in a randomized controlled trial. J Clin Epidemiol 2006;59:404-11.

13. Shajrawi A, Granat M, Jones I, et al. Physical activity and cardiac self-efficacy levels during early recovery after acute myocardial infarction: A Jordanian study. J Nurs Res 2020;29:e131.

14. Ashford S, Edmunds J, French DP. What is the best way to change self-efficacy to promote lifestyle and recreational physical activity? A systematic review with meta-analysis. Br J Health Psychol 2010;15:265-88.

15. Bandura A. Personal and collective efficacy in human adaptation and change. Adv Psychol Sci 1998;1:51-71.

16. Anselm Strauss JMC. Basics of qualitative research. Thousand Oaks: Sage Publications, Inc.; 1998.

17. Williams SL, French DP. What are the most effective intervention techniques for changing physical activity self-efficacy and physical activity behaviour - And are they the same? Health Educ Res 2011;26:308-22.

18. Ferrier S, Dunlop N, Blanchard C. The role of outcome expectations and self-efficacy in explaining physical activity behaviours of individuals with multiple sclerosis. Behav Med 2010;36:7-11.

19. Bandura A. Social foundations of thought and action. Englewood Cliffs: Prentice Hall; 1986.

20. Chodzko-Zajko WJ, Proctor DN, Fiatarone Singh MA, et al. Exercise and physical activity for older adults. Med Sci Sports Exerc 2009;41:1510-30.

21. Pickett K, Kendrick T, Yardley L. "A forward movement into life": A qualitative study of how, why and when physical activity may benefit depression. Ment Health Phys Act 2017;12:100-9.

22. Irmak Z, Fesci H. Effects of nurse-managed secondary prevention program on lifestyle and risk factors of patients who had experienced myocardial infarction. Appl Nurs Res 2010;23: 147-52.

23. Uysal H, Özcan Ş. The effect of individual training and counselling programme for patients with myocardial infarction over patients' quality of life. Int J Nurs Pract 2012;18:445-53.

24. Harbman P. The development and testing of a nurse practitioner secondary prevention intervention for patients after acute myocardial infarction: A prospective cohort study. Int J Nurs Stud 2014;51:1542-56.

25. Lau-Walker M, Landy A, Murrells T. Personalised discharge care planning for postmyocardial infarction patients through the use of the Personalised Patient Education Protocol - implementing theory into practice. J Clin Nurs 2016;25:1292-300.

26. Vibulchai N, Thanasilp S, Preechawong S. Randomized controlled trial of a self-efficacy enhancement program for the cardiac rehabilitation of Thai patients with myocardial infarction. Nurs Health Sci 2016;18:188-95. 